\title{
Growth of Gold Nanorods and Bipyramids Using CTEAB Surfactant
}

Xiaoshan Kou, Shuzhuo Zhang, Chia-Kuang Tsung, Man Hau Yeung, Qihui Shi, Galen D. Stucky, Lingdong Sun, Jianfang Wang, and Chunhua Yan

\section{Supporting Information}
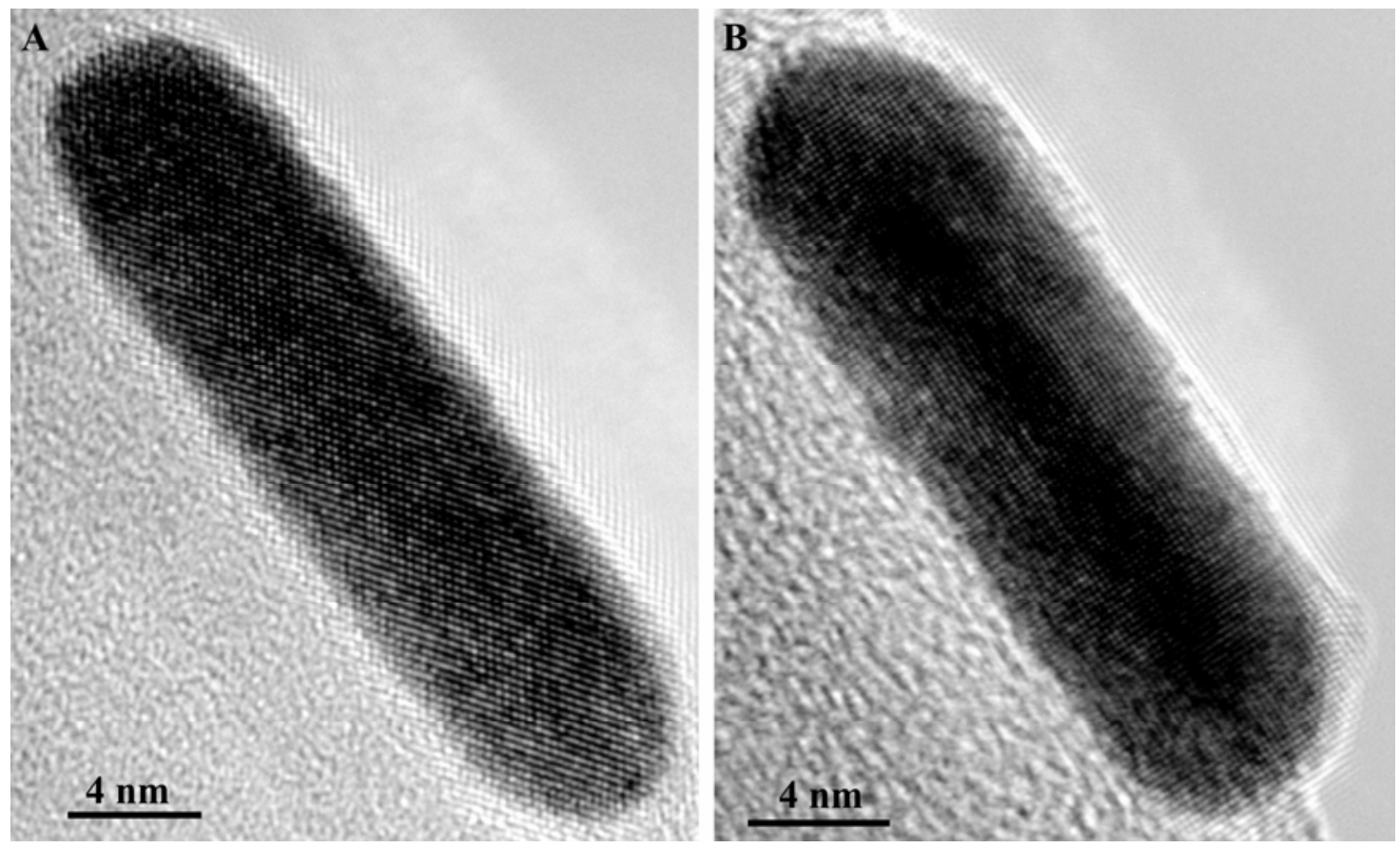

Figure S1. (A) HRTEM image of one gold nanorod oriented in the [110] direction. The aspect ratio of the rod is 3.8. (B) HRTEM image of one gold nanorod oriented in the [100] direction. The aspect ratio of the rod is 3.2. The rods were synthesized with $0.032 \mathrm{~mL}$ of the CTEAB-stabilized seed solution. 


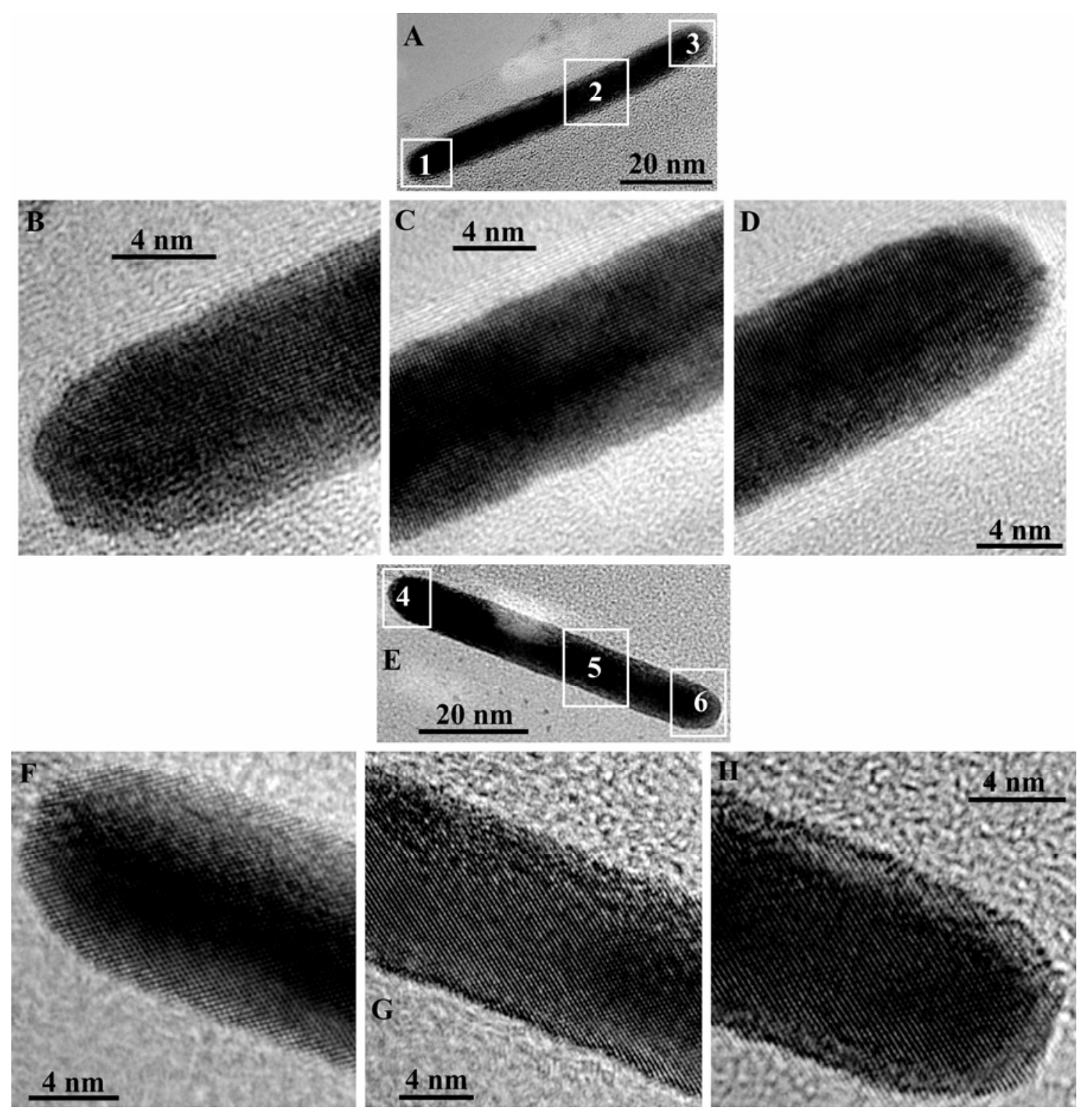

Figure S2. (A) LMTEM image of one gold nanorod oriented in the [100] direction. The aspect ratio of the rod is 9.6. (B), (C), and (D) HRTEM images recorded from the boxed areas (1), (2), and (3) shown in (A), respectively. (E) LMTEM image of one gold nanorod oriented in the [110] direction. The aspect ratio of the rod is 7.6. (F), $(\mathrm{G})$, and (H) HRTEM images recorded from the boxed areas (4), (5), and (6) shown in (E), respectively. The rods were synthesized with 0.0065 $\mathrm{mL}$ of the CTEAB-stabilized seed solution. 

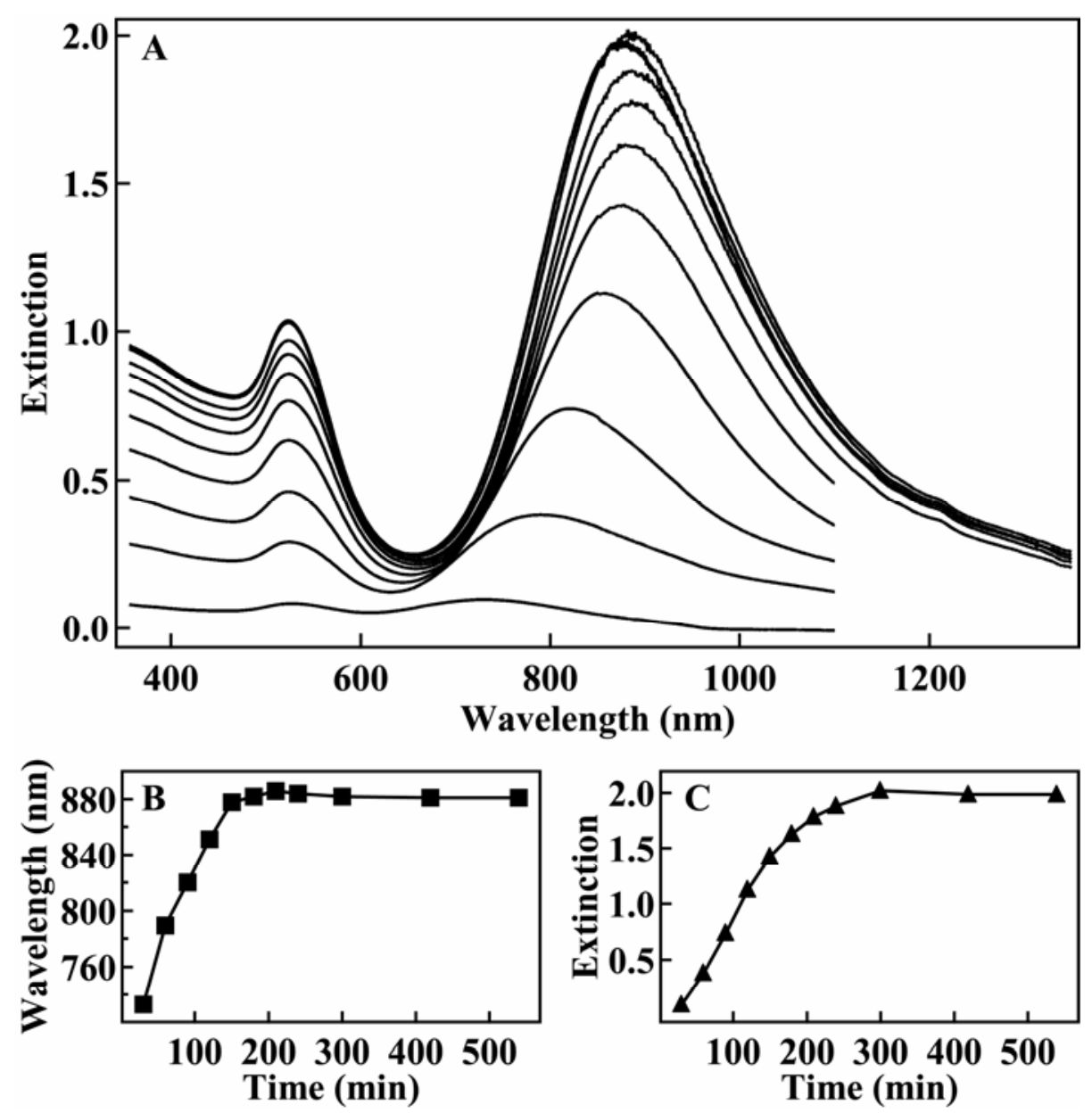

Figure S3. (A) Extinction spectra of the nanorod growth solution as a function of time after the addition of $0.032 \mathrm{~mL}$ of the CTEAB-stabilized seed solution. (B) Temporal evolution of the LPW during growth. (C) Temporal evolution of the longitudinal plasmon peak extinction during growth. The data points in (B) and (C) were extracted from the curves shown in (A). 

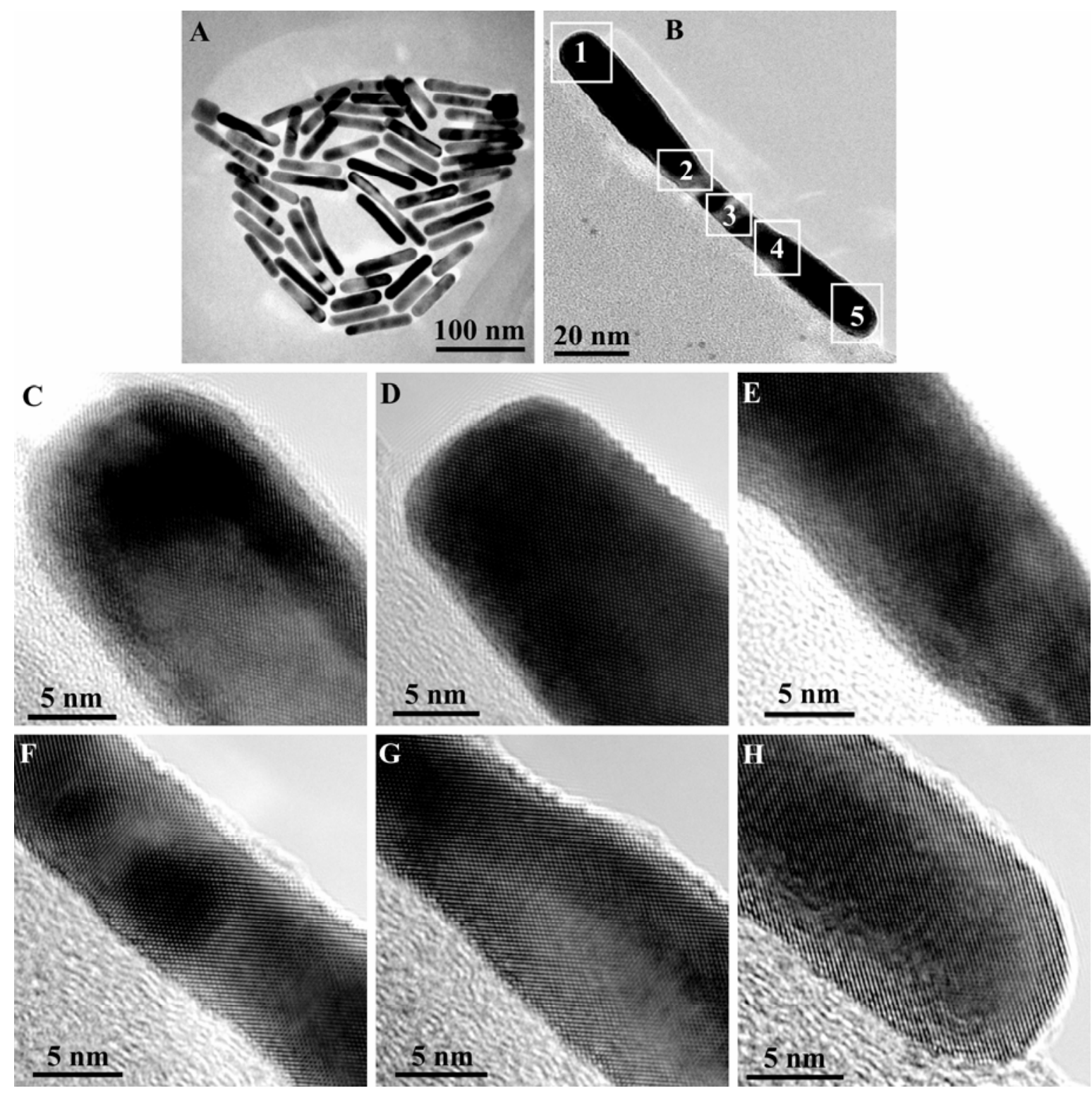

Figure S4. (A) LMTEM image of the gold nanorods obtained from the two-step growth process. For the first-step growth, $0.0065 \mathrm{~mL}$ of the CTEAB-stabilized seed solution was used. (B) TEM image of a single nanorod obtained from the two-step growth process as in (A). It is oriented along the [110] zone axis. (C) HRTEM image recorded from the boxed area (1) shown in (B). (D) HRTEM image recorded from the boxed area (1) shown in (B) after electron beam irradiation for 0.5 h. (E), (F), (G), and (H) HRTEM images recorded from the boxed areas (2), (3), (4), and (5) shown in (B), respectively, after electron beam irradiation. 

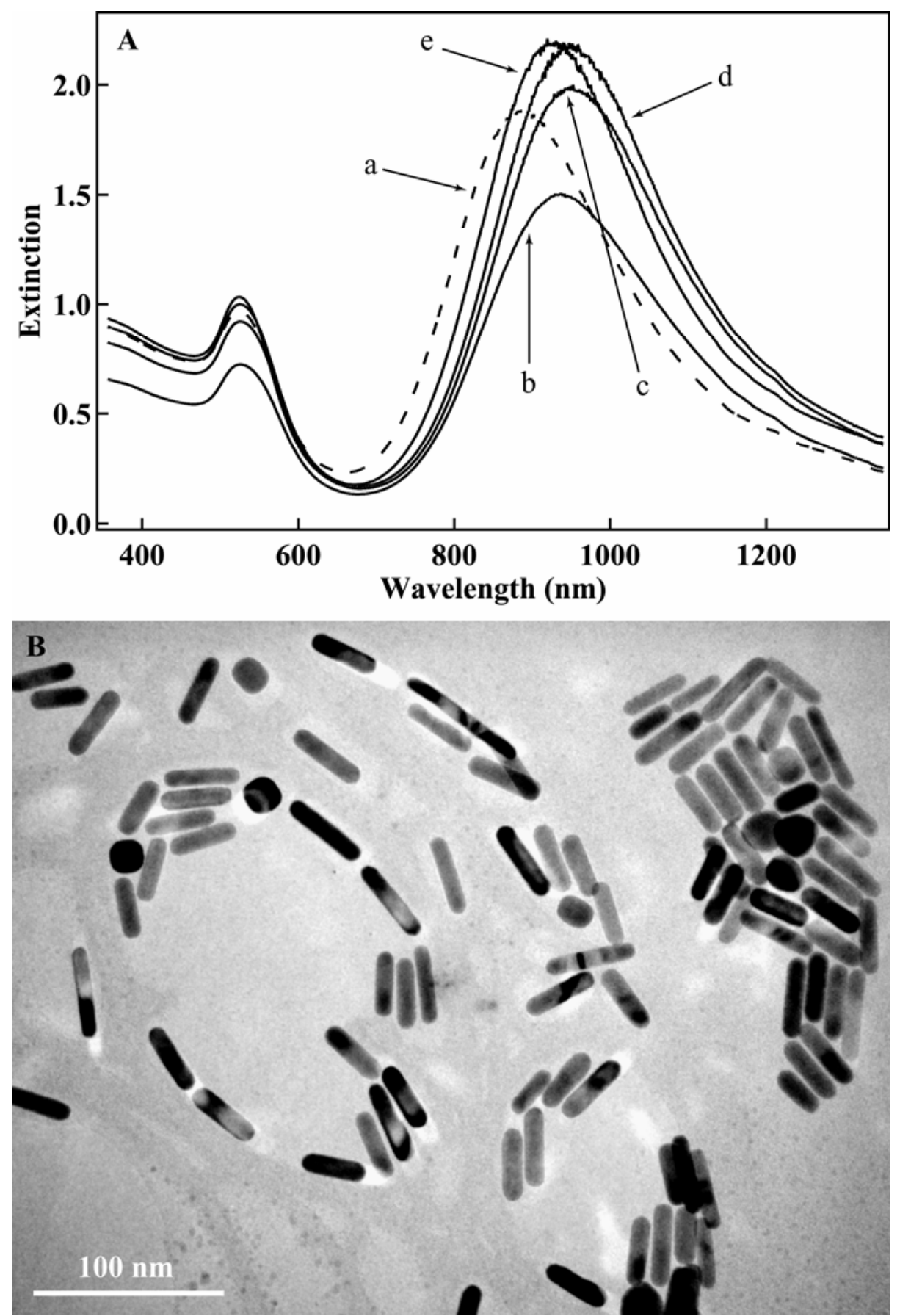

Figure S5. (A) Extinction spectra taken as a function of time after the growth solution was added to a gold nanorod solution at a volume ratio of 1 to 1 for the second-step growth. The nanorod solution was taken directly from the first-step growth, for which $0.032 \mathrm{~mL}$ of the CTEAB-stabilized seed solution was used, and the growth time was $4.5 \mathrm{~h}$. The spectrum (a) (dashed line) was taken right before the second-step growth. The spectra (b), (c), (d), and (e) were taken $60 \mathrm{~min}, 180 \mathrm{~min}, 270 \mathrm{~min}$, and $12 \mathrm{~h}$, respectively, after the addition of the growth solution for the second-step growth. (B) LMTEM image of the gold nanorods obtained from the two-step growth process. 

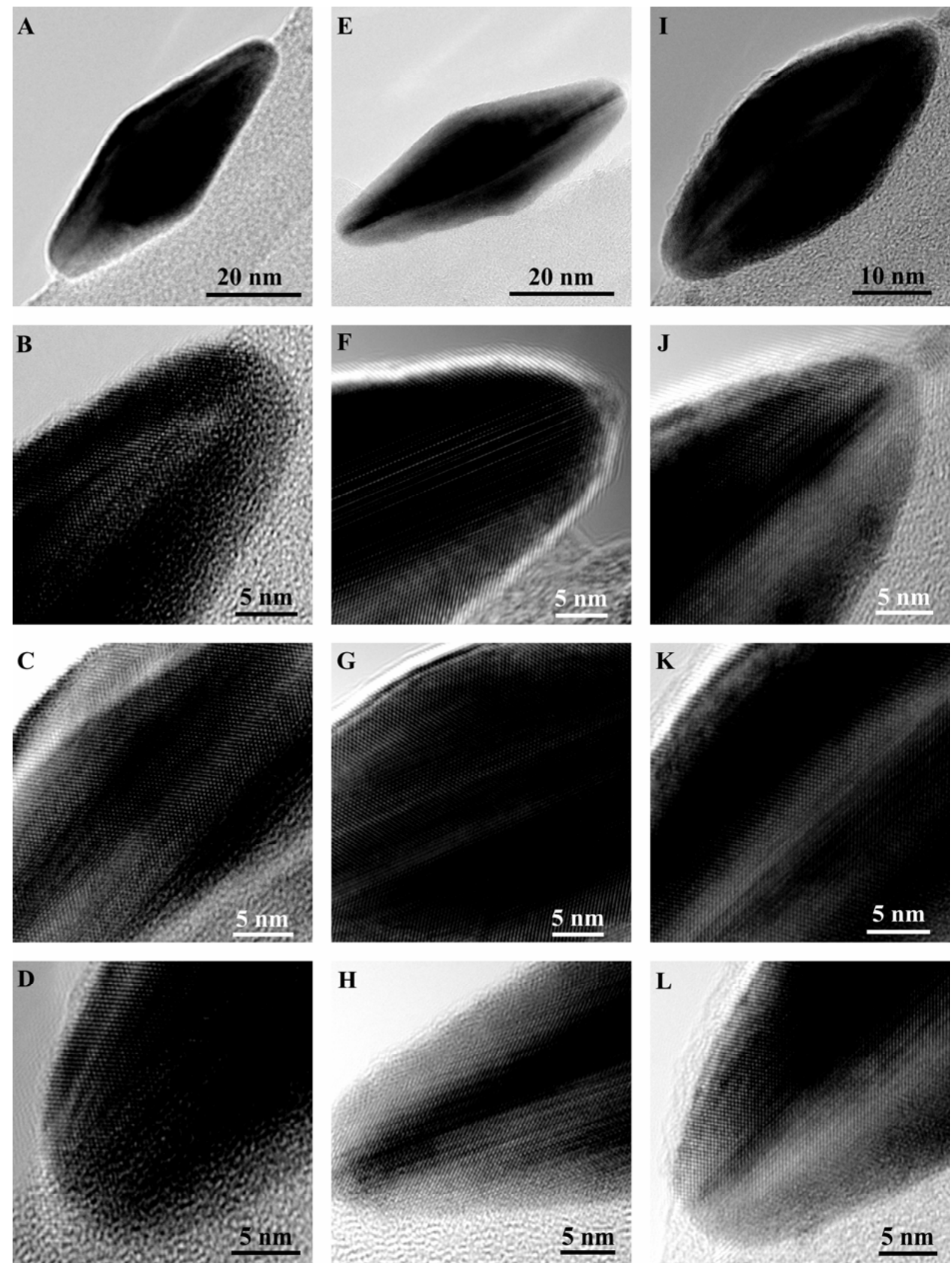

Figure S6. (A), (E), and (I) LMTEM images of three individual gold bipyramids. (B), (C), and (D) HRTEM images of the different areas of the bipyramid shown in (A), (F), (G), and (H) 
HRTEM images of the different areas of the bipyramid shown in (E). (J), (K), and (L) HRTEM images of the different areas of the bipyramid shown in (I). The bipyramids were grown with $0.12 \mathrm{~mL}$ of the citrate-stabilized seed solution. 